\title{
A new framework for high-resolution pedestrian data processing using rule-based algorithms and real-time alarm systems
}

\author{
Michael Moos ${ }^{1}$, Basil Vitins ${ }^{2}$, Mirwais Tayebi ${ }^{3}$, \\ Lukas Gamper ${ }^{4}$, Julia Wysling ${ }^{5}$, Uri Schtalheim ${ }^{6}$ \\ 1,2,3,4,5,6 ASE AG \\ Zurich, Switzerland \\ michael.moos@ase.ch
}

\begin{abstract}
Pedestrian flows and densities have increased in recent years within transport-related public facilities such as train stations, as well as in private buildings such as shopping centers, event halls or convention centers. Increasing flows and high densities often raise comfort, safety, operational and delay issues; and therefore, require pedestrian flow optimization, intervention or even revised regulation. Recent technological advances enhanced pedestrian sensing; however, they disregard adaptive data capture, processing, and strategic communication within reasonable time, or real-time, such as tactic occupancy or density alarms trigger rules. Content of this research is twofold. First, new data capturing and processing advances of recent technological developments are combined in an integral software and hardware-based framework. Second, applied methods highlight projects and experiences on both pedestrian research and on existing and operating pedestrian facilities. Based on the described, two-sided approach, proposed framework is able to fulfil high safety and comfort standards of facilities such as train stations, retail facilities or event halls. In this research, past semi-automatic video analysis processing of pedestrian behavioral studies is replaced with combined sensor and data processing system within proposed framework. In train stations of major operators, real-time pedestrian observation increases safety levels on station platforms. Tactic algorithms and alarm trigger schemes enable on-time surveillance, e.g. at overcrowded floor levels in shopping centers for escalator or door closure. Sensor data is used to train models for underpass pedestrian flow regarding path choice and fundamental diagram. In retail, queue length, trajectory analysis and floor occupancy are determined for economic, comfort as well as safety evaluation. Using trajectory classification, movement and dwell time is analyzed for staff and visitors separately (see Figure 1).
\end{abstract}

Keywords:

Video Analysis, Realtime Monitoring, Alarming, Model Calibration, Trajectory Classification

\section{Introduction}

Recent population growth in many countries increase densities in public spaces such as public transportation and its demands; e.g. London already has special schemes for their underground lines such as temporarily closed stations for boarding passengers. Technological developments have enabled sensing for precise movement captures, including fast data processing such as image recognition. Infrastructure operators require reliable sensing technologies and highly aggregated and selected data to provide security, comfort and functionality to their infrastructure. Infrastructure usage increases and demand gets closed to the limits.

There is a cap recognized between sensing technologies with their large and raw data output, and corresponding evaluation and application requiring aggregated data for end users. The goal of this study is therefore a generic framework proposition to cover such high and specialized requirements. The framework is adaptive in areas, environments (indoor, outdoor) and relies on generic data capturing, processing and visualization chain. New advance in sensing can therefore be used for operation purposes. 


\section{Data Capturing}

Various technologies capture pedestrian movements. Most relevant technologies rely on video analysis, laser sensing, WiFi and Bluetooth signal detection. Video and laser rely on light waves and can capture precise images. In comparison to image processing, WiFi capturing is less adequate, since often only about $20 \%$ and $40 \%$ of all persons carry at least one active WIFI device. For indoor applications, video-based sensors are the most established while in outdoor applications, laser-based sensors have advantages, especially with little ambient light or weather-related influence. For indoor and outdoor, sensors are usually mounted on the ceiling for vertical detection to increase precision. This strategy is important, especially in situations with high densities where pedestrians are hidden between each other (example see Figure 1).

Pedestrian sensing can mainly be divided into 3 categories. Initially, pedestrian analysis was limited in counting persons crossing a virtual counting line. Later, area-based measurement evolved for advanced evaluations. By defining not just a virtual line, but a virtual area, many more applications became possible like waiting time or queue length in certain, user-defined areas (rectangles). In recent years, sensors where developed which were capable to record trajectories, enabling precise person tracking.

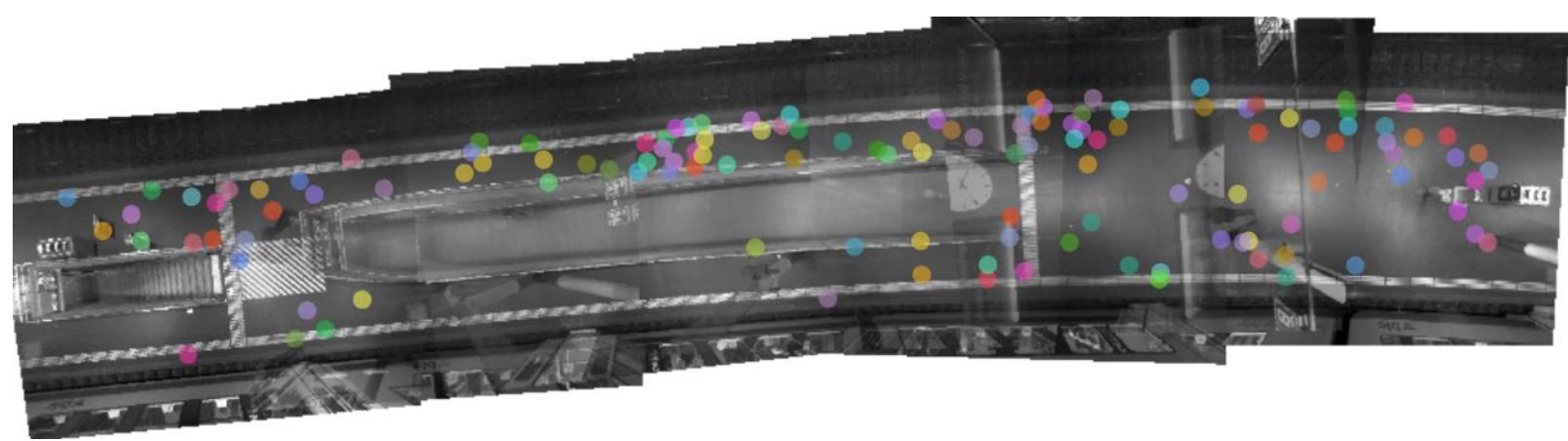

Figure 1. Example top-down view of a video based pedestrian sensor. The image shows waiting travelers on a swiss railway platform. The goal of this application is the evaluation of passovers of the white barrier line.

\section{Applications}

\subsection{Real-Time Monitoring}

In real-time monitoring, a dashboard with all necessary information is often used for analysis. Dashboards are synchronized with live measurements from sensor protocols. A parallel running, permanent system control is essential for reliability. Notifications must appear immediately in case of component failure or irregularities. Depending on the sensing area, dangerous pedestrian situations (e.g. high densities) not only require a dashboard for overview but also the possibility of alarming staff using text messages or internet capable devices. In recent years, many such devices have been released like smart light bulbs or speakers. Pedestrian data are essential to many IoT applications requiring efficient data processing. These not only apply on security but also the signaling for staff, when large queues arise in sales. Using rule-based algorithms, customized thresholds are defined for alarm and notification triggering.

\subsection{Analytics}

Major challenge in analytics includes information extraction and processing from large-scale raw data. Often, aggregation becomes essential regarding time and space. While aggregation is trivial for frequency-based information (e.g. time series), aggregation of combined space and time-related information is more demanding (e.g. dwell time) and often not well-defined. Trajectory-based data even allow many more possibilities like trajectory classification and origin-destination extraction. Figure 2 
shows an example of trajectory classification. Movement and dwell time is analyzed separately for staff and visitors, leading to new insights at the planning of store layouts. In this example, trajectories were classified regarding the position of origin and termination.
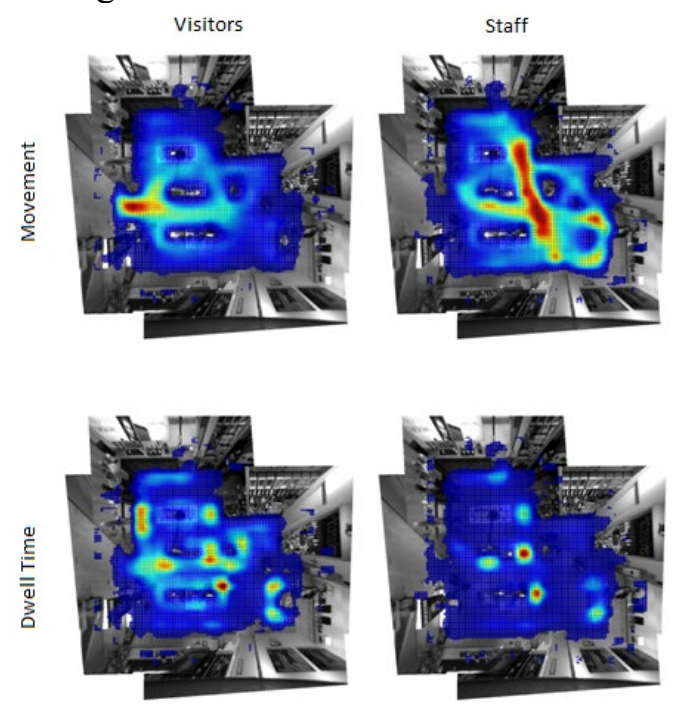

Figure 2. Using trajectory classification, movement and dwell time is analyzed for staff and visitors separately.

\subsection{Flow Models}

Data monitoring for pedestrian movement can be costly for complete floor area coverage. Necessary measurements can be drastically reduced when adding pedestrian flow models. This approach is most effective in transport applications like train stations, where shortest path assumptions apply. In all situations, high-resolution pedestrian data is crucial in model calibration. Data capturing and model calibration are used iteratively for high reliability in pedestrian flow prediction.

\section{Conclusion}

In the previous decade, image processing and sensing technologies were the most challenging subject in pedestrian movement analysis. Today, these issues have mostly been solved. With that, the extraction of information from data has become the most essential part in the area.

The proposed framework closes existing gaps between the raw data output of high-end sensing technologies and the required information by the wide range of end users. Key strategy is the two-sided approach of real-time monitoring for instant notification and intelligent data aggregation and visualization for long term analytics. With the proposed framework, a wide range of possible applications are covered not only for researchers but also management.

\section{References}

[1] J. Thurau, J. Van den Heuvel, M. Ofwegen, N. Keusen and S. Hoogendoorn „Influence of pedestrian density on the use of the danger zone at platforms of train stations", Conference on Traffic and Granular Flow, Washington DC, July 2017.

[2] D. Helbing and A. Johansson "Pedestrian, Crowd and Evacuation Dynamics", Encyclopedia of Complexity and Systems Science, vol. 16, pp. 6476-6495, 2010.

[3] T. Kretz, "Pedestrian traffic: on the quickest path", J.Stat. Mech. Theor. Exp., vol. 03, pp. 1-13, 2009.

[4] A. Johansson, D. Helbing and P.K. Shukla, "Specification of the social force pedestrian model by evolutionary adjustment to video tracking data", Adv. Compl. Syst., vol. 10, pp. 271-288.

[5] S. Buchmueller and U. Weidmann, Parameters of pedestrians, pedestrian traffic and walking facilities, Schriftenreihe, vol. 132, ETH Zurich, 2007.

[6] D. Duives, W. Daamen and S. Hoogendoorn "Quantification of the level of crowdedness for pedestrian movements", Physica A Stat. Mech. Appl., vol. 427, June 2015. 